\title{
Statistical properties of local AGNs based on the INTEGRAL/IBIS 7-year all-sky hard X-ray survey
}

\section{Sergey Sazonov*}

Space Research Institute of the Russian Academy of Sciences, Moscow, Russia

E-mail: sazonoveiki.rssi.ru

\section{Eugene Churazov}

Max-Planck Institute for Astrophysics, Germany

and

Space Research Institute of the Russian Academy of Sciences, Moscow, Russia E-mail: churazov@mpa-garching.mpg.de

\section{Roman Krivonos}

Max-Planck Institute for Astrophysics, Germany

and

Space Research Institute of the Russian Academy of Sciences, Moscow, Russia E-mail: krivonos@mpa-garching.mpg.de

\section{Mikhail Revnivtsev}

Space Research Institute of the Russian Academy of Sciences, Moscow, Russia E-mail: revnivtsevaiki.rssi.ru

\section{Rashid Sunyaev}

Max-Planck Institute for Astrophysics, Germany

and

Space Research Institute of the Russian Academy of Sciences, Moscow, Russia E-mail: sunyaev@mpa-garching.mpg.de

8th INTEGRAL Workshop "The Restless Gamma-ray Universe"

September 27-30 2010

Dublin Castle, Dublin, Ireland

\footnotetext{
* Speaker.
} 


\section{Introduction}

The INTEGRAL observatory continues to provide high quality scientific data after 8 years of being in orbit. INTEGRAL performs pointed observations of miscellaneous targets in the sky and (less frequently) scans of extended sky regions; as a result virtually the whole sky has been covered by observations over the course of the mission. The full set of INTEGRAL data thus represents a serendipitous, all-sky survey in the hard X-ray band, which can be used for both Galactic and extragalactic population studies.

The INTEGRAL survey is characterized by strongly inhomogeneous exposure and hence substantially variable limiting flux for source detection over the sky. Although this can present a significant problem for certain (in particular, volume limited) studies, as compared to dedicated surveys (such as the planned SRG/eRosita X-ray all-sky survey), it may also be regarded as an advantage, since one essentially has a collection of surveys of different depths. Moreover, there is potential synergy between the INTEGRAL survey and a similar serendipitous survey carried out by the Swift satellite since the end of 2004. Indeed, the INTEGRAL survey is stronlgy focused on the \pm 20 degree strip around the Galactic plane, which is essentialy avoided by Swift; the latter, however, has covered the rest of the sky with better sensitivity [8].

One of the best uses of the INTEGRAL all-sky survey is exploring the local population of active galactic nuclei (AGNs). Our team previously did such a research based on the catalog of sources detected by IBIS/ISGRI in the 17-60 keV energy band before mid-2006 [1], hereafter referred to as the 3.5-year catalog. This catalog includes 130 previously known and newly discovered AGNs, 94 of which are at least $5 \sigma$ detections on the time-averaged IBIS/ISGRI map of the sky. We used the subsample of 66 non-blazar AGNs located at $|b|>5^{\circ}$ to study various properties of the local $(z \lesssim 0.1)$ population of AGNs:

- We measured the hard X-ray (17-60 keV) luminosity function (LF) and cumulative luminosity density of local AGNs with $L_{17-60 \mathrm{keV}}>10^{40} \mathrm{erg} \mathrm{s}^{-1}$ [5].

- We verified that the ratio of the numbers of absorbed and unabsorbed AGNs in the local Universe decreases with increasing luminosity [5].

- We found that strongly obscured AGNs (almost or strictly Compton thick objects, with $N_{\mathrm{H}}>$ $10^{24} \mathrm{~cm}^{-2}$ ) are a small, $\sim 10 \%$, fraction of hard X-ray selected AGNs [7].

- We stacked the AGN spectra in the 15-300 keV energy band, weighting them according to the AGN LF, and thus derived the cumulative hard X-ray spectrum of the local population of AGNs. Its curved shape is consistent with that expected for sources that make up the cosmic $\mathrm{X}$-ray background (CXB) [6].

Recently, our team has issued an updated all-sky catalog of sources, detected after almost 7 years (until July 2009) of INTEGRAL observations [3]. Compared to the previous version, this 7year catalog is characterized by the following advantages: i) more than doubled total exposure, ii) better method of source detection in crowded fields [2], and iii) better coverage of the extragalactic sky. As a result, the new sample of AGNs at $|b|>5^{\circ}$ includes 15 blazars and 147 non-blazar AGNs. The latter number represents more than a factor of 2 increase with respect to the 3.5-year catalog. 


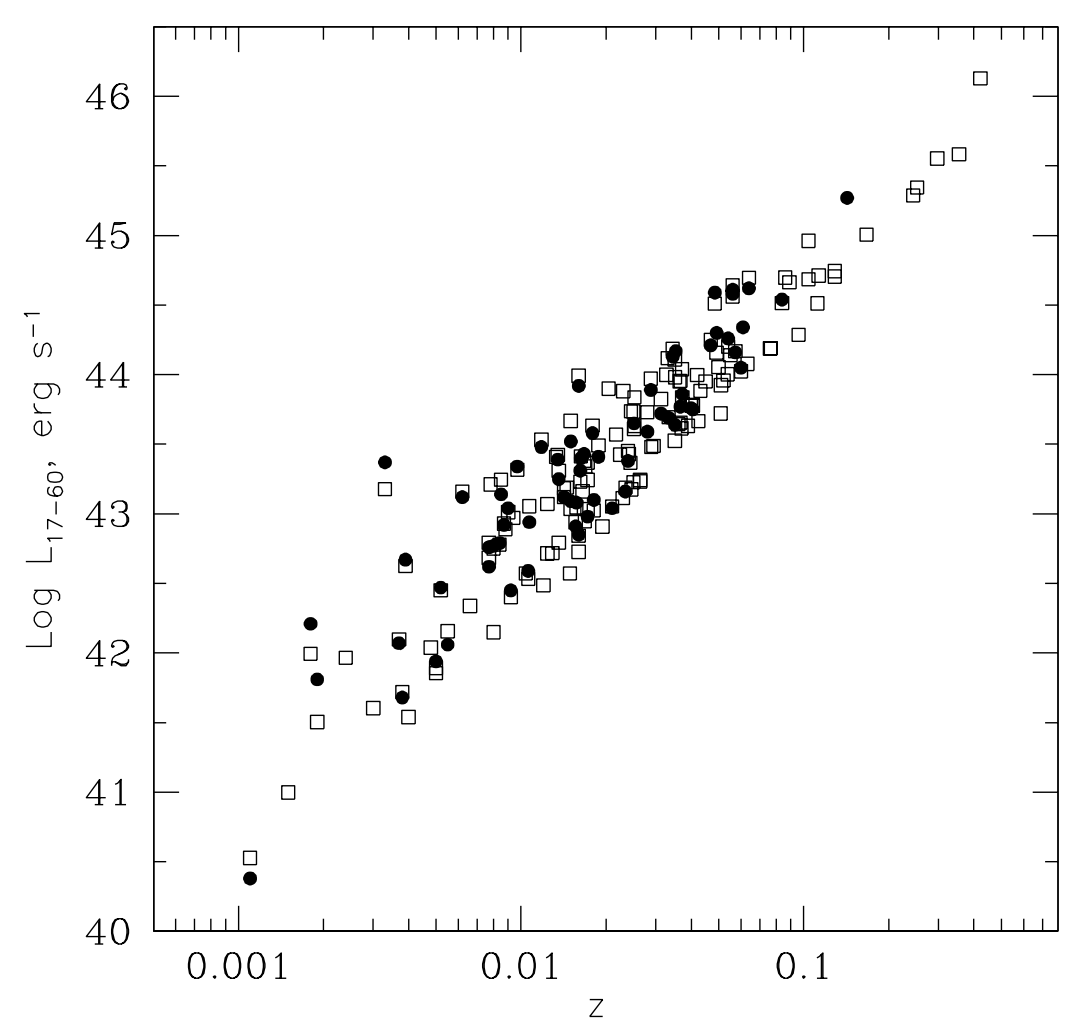

Figure 1: Hard X-ray luminosity vs. redshift for non-blazar AGNs located at $|b|>5^{\circ}$ from the 3.5-year (filled circles) and 7-year (open squares) catalogs. Note that we exclude the $|b|<5^{\circ}$ region - the "zone of avoidance" - from the analysis below because of the remaining identification incompleteness there.

Fig. 1 shows the distribution of the non-blazar AGNs in the redshift-luminosity plane. One can see that the most noticeable improvements pertain to the regions of lowest $\left(40.5<\log L_{17-60 \mathrm{keV}}<\right.$ $42.5)$ and highest $\left(\log L_{17-60 \mathrm{keV}}>44.5\right)$ luminosities, which were poorly populated in the 3.5-year catalog.

\section{Results}

Here we present a number of preliminary statistical results for AGNs based on the 7-year catalog.

\subsection{Hard X-ray luminosity function}

Due to the substantially improved sampling of both the low- and high-luminosities regimes, we can now measure the hard X-ray luminosity function with significantly better accuracy. The new LF is shown in the left panel of Fig. 2. It can be fit by a smoothly broken power law (the standard model in AGN studies) with slopes of the low- and high-luminosity ends of $0.81 \pm 0.11$ and $2.28 \pm 0.14$, respectively, and a break luminosity $\log L_{*}=43.56 \pm 0.18$ (assuming $H_{0}=72 \mathrm{~km} \mathrm{~s}^{-1} \mathrm{Mpc}^{-1}$ ). Integration over the LF yields the cumulative luminosity density of local AGNs in the 17-60 keV energy band: $(1.46 \pm 0.18) \times 10^{39} \mathrm{erg} \mathrm{s}^{-1} \mathrm{Mpc}^{-3}$. These values are consistent with but more accurate by a factor of $\sim 2$ than those based on the 3.5-year catalog [5]. 


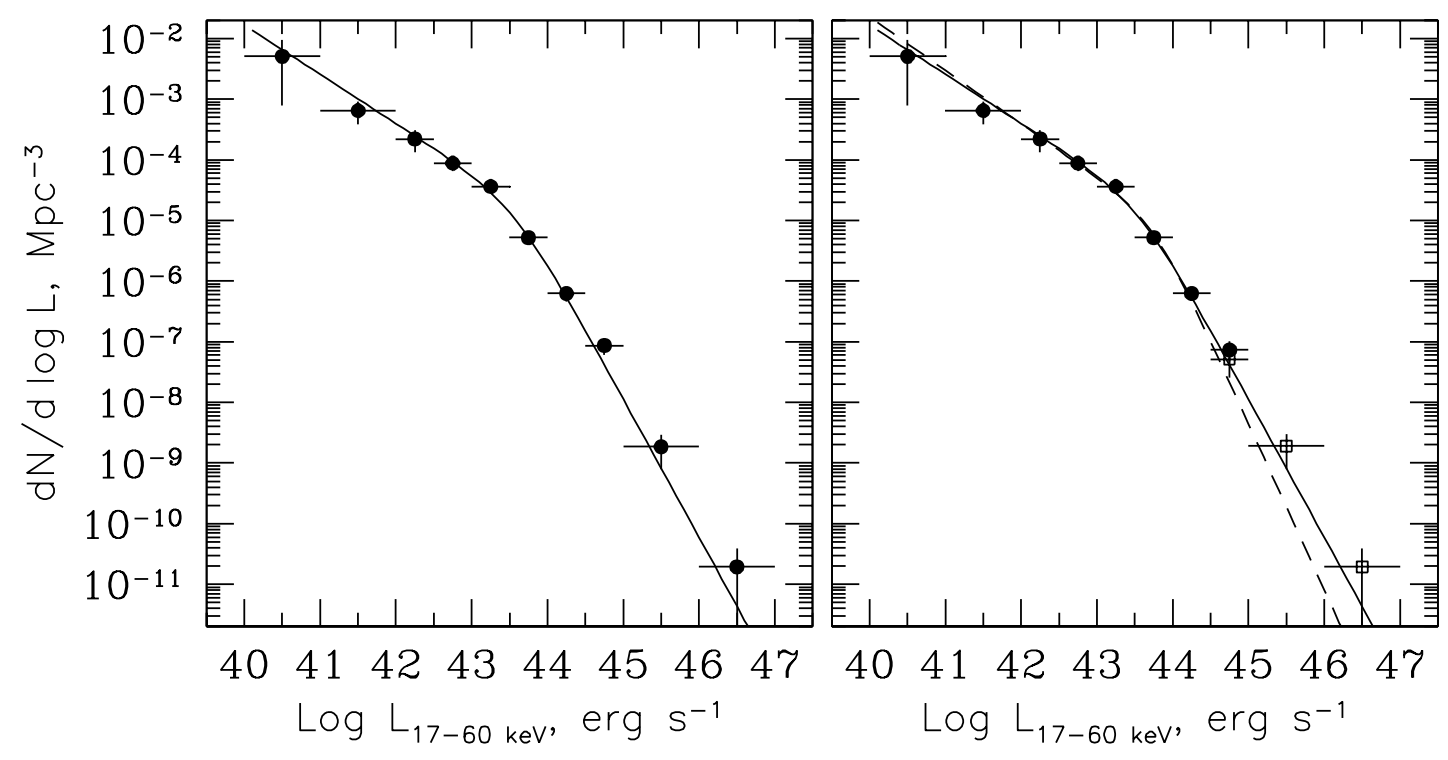

Figure 2: Left: Binned luminosity function of AGNs in the 17-60 keV energy band and its best fit by a broken power law (see parameter values in the main text). Right: Luminosity function of AGNs at $z<0.1$ (solid circles) and $0.1<z<1$ (empty squares). The solid and dashed lines are the best fitting models of all and the $z<0.1$ data, respectively.

The highest luminosity sources in our sample have significant redshifts, and so one may worry if the derived LF really represents the local AGN population. To address this question, we show in the right panel of Fig. 2 the LFs of AGNs at $z<0.1$ and $0.1<z<1$ (135 and 12 objects, respectively). We see good agreement at $\log L_{17-60 \mathrm{keV}}=44.5$, where both LFs overlap. However, fitting the $z<0.1$ data by a broken power-law model indicates that the slope of the local LF may be somewhat steeper $(2.74 \pm 0.37)$ than is implied by the total data set, including $z>0.1$ objects. This result is however only $\sim 1 \sigma$ significant.

\subsection{Fraction of obscured AGNs}

The next key question is obscuration in AGNs. The left panel of Fig. 3 shows the distribution of absorbing column densities for our AGNs. These estimates have been adopted from the literature and our analysis of data from different X-ray telescopes. (see Sazonov et al., in preparation). Based on the 7-year catalog, $54 \%$ of AGNs can be classified as X-ray absorbed $\left(\log N_{\mathrm{H}}>22\right)$. This result is consistent with previous estimates based on smaller samples of INTEGRAL detected AGNs [5, 4]. We also confirm that the observed fraction of heavily obscured objects $\left(\log N_{\mathrm{H}}>24\right)$ is low, $\sim 8 \%$. Finally, we confirm that there is a strong trend of decreasing relative abundance of X-ray absorbed AGNs with increasing luminosity (the right panel of Fig. 3).

\subsection{Stacked spectra}

Since the detection significance in not high for most of our sources, it is practically impossible to study IBIS/ISGRI spectra on a source by source basis. However, we can stack individual spectra 

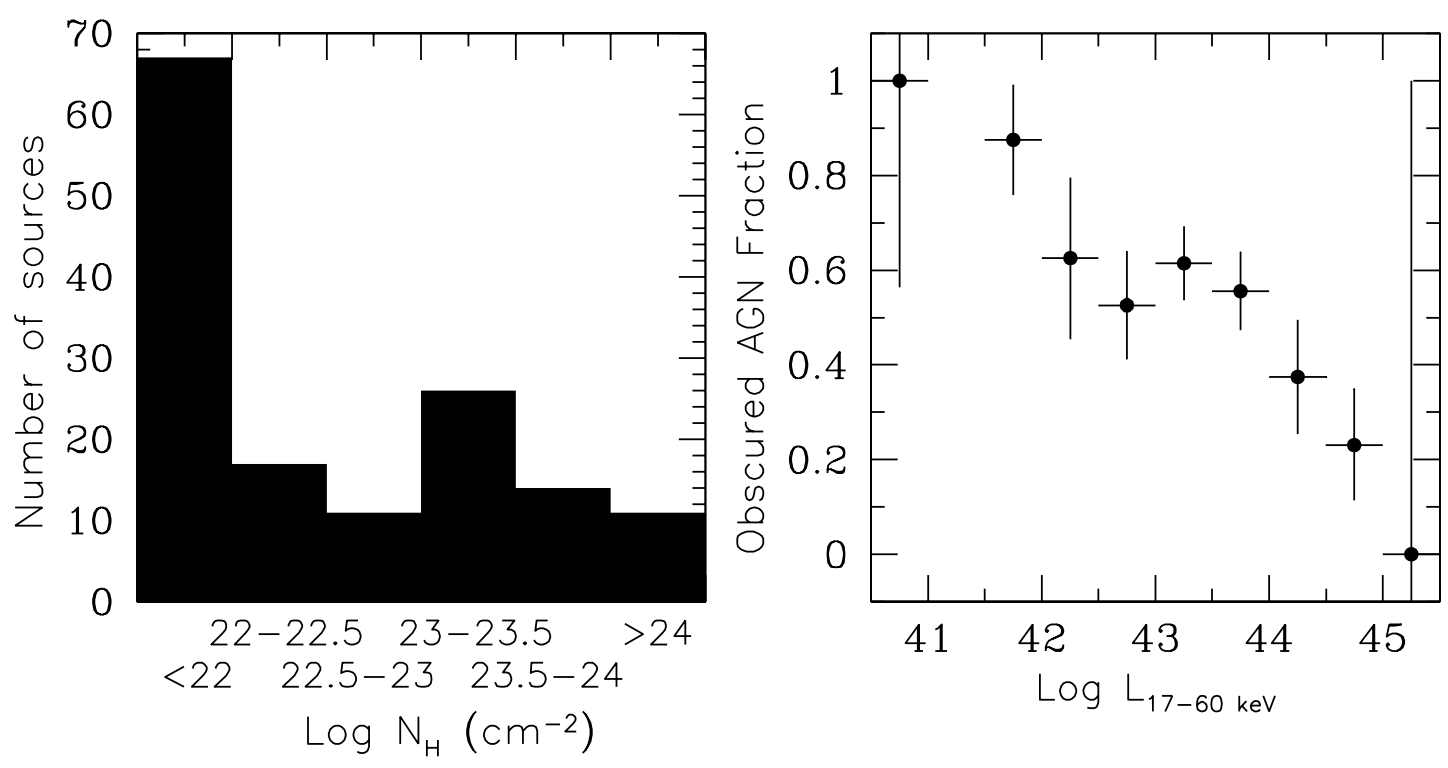

Figure 3: Left: Histogram of X-ray absorbing column densities of AGNs from the 7-year catalog. Right: Fraction of obscured $\left(\log N_{\mathrm{H}}>22\right)$ objects as a function of luminosity.

to study the spectral energy distribution of local AGNs. In the left panel of Fig. 4 we show the stacked 17-194 keV spectra of three subsamples of AGNs: those detected with significance of $5-15 \sigma$ (120 objects), $15-30 \sigma$ (20 objects), and $>30 \sigma$ (11 objects). In all three cases there is an indication that the spectrum bends downward at energies higher that $\sim 70 \mathrm{keV}$. Fitting the spectra by a power law with an exponential cutoff yields photon indices $\sim 1.5-1.8$, with the cutoff best detected for the $>30 \sigma$ group, where $E_{f}=240 \pm 30 \mathrm{keV}$.

Stacking individual spectra with weights determined by the space densities of AGNs of given luminosity, we can obtain the spectral volume emissivity of the local AGN population (see [6] for the details of this analysis). The so derived spectrum is presented in the right panel of Fig. 4. The relatively poor quality of the spectrum is owing to the fact that it is by construction dominated by contributions from just a few low-significance sources. A cutoff power law with $\Gamma=1.56$ and $E_{f}=120 \mathrm{keV}$ gives a somewhat better fit to the spectrum than a simple power law with $\Gamma=1.93$. However, the significance of detection of the cutoff is very low. We are now working on removing some remaining systematic effects and adding a 194-290 keV point to the spectra shown in Fig. 4, which should lead to a better understanding of the spectral behavior of AGNs at high energies.

\section{Conclusions}

The preliminary results obtained based on the 7-year AGN catalog confirm and strengthen our previous findings from the 3.5-year catalog:

- The local hard X-ray LF is now known to within 10-20\% over several orders of magnitude, $41 \lesssim \log L_{17-60 \mathrm{keV}} \lesssim 45$. It can therefore serve as a reliable zero-redshift template for studying the evolution of the AGN LF over the cosmic time - the phenomenon of downsizing - 

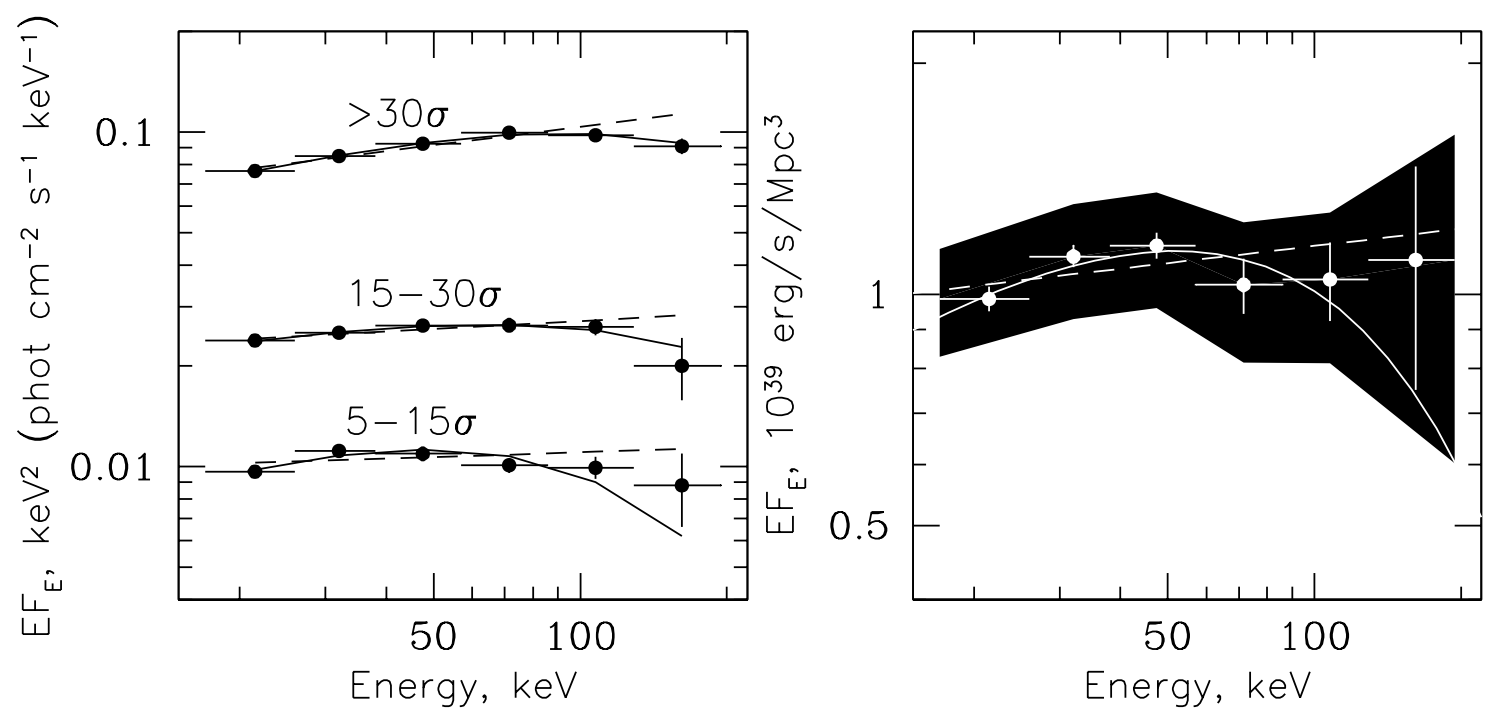

Figure 4: Left: Stacked spectra of AGNs divided in three groups according to detection significance. Right: Spectral volume emissivity of the local population of AGNs (data points with uncertainties indicated by error bars and the shaded region) and the best fits by a power law and by a power law with exponential cutoff.

using data of currently available and upcoming (NuSTAR, SRG) deep X-ray and hard X-ray surveys.

- The phenomenon of decreasing ratio of numbers of obscured and unobscured AGNs with increasing luminosity has been revealed better than before. It probably indicates the decreasing effective solid angle of the dusty torus - the key element of AGN unification schemes. The physical cause of this behavior still needs to be understood.

- The derived stacked spectra are broadly consistent with the shape of AGN spectra required to explain the spectrum of the cosmic X-ray background, although the significance of detection of a high-energy cutoff is still low.

The INTEGRAL observatory continues to provide great data for studying the local population of AGNs. As more data are accumulated over the next several years, we may expect to achieve a significant improvement first of all in the average AGN spectrum. One should also think about a combined analysis of AGN statistics provided by INTEGRAL and Swift.

The research made use of grants RFBR 09-02-00867a and NSH-5069.2010.2 and RAS programs P-19 and OFN-16. SS acknowledges the support of the Dynasty Foundation.

\section{References}

[1] Krivonos, R., Revnivtsev, M., Lutovinov, A., Sazonov, S., Churazov, E., \& Sunyaev, R. 2007, Astron. Astrophys., 475, 775

[2] Krivonos, R., Revnivtsev, M., Tsygankov, S., Sazonov, S., Vikhlinin, A., Pavlinsky, M., Churazov, E., \& Sunyaev, R. 2010, Astron. Astrophys., 519, A107 
[3] Krivonos, R., Tsygankov, S., Revnivtsev, M., Grebenev, S., Churazov, E., \& Sunyaev, R. 2010, Astron. Astrophys., 523, A61

[4] Malizia, A., Stephen, J. B., Bassani, L., Bird, A. J., Panessa, F., \& Ubertini, P. 2009, MNRAS, 399, 944

[5] Sazonov, S., Revnivtsev, M., Krivonos, R., Churazov, E., \& Sunyaev, R. 2007, Astron. Astrophys., 462, 57

[6] Sazonov, S., Krivonos, R., Revnivtsev, M., Churazov, E., \& Sunyaev, R. 2008, Astron. Astrophys., 482, 517

[7] Sazonov, S., Revnivtsev, M., Burenin, R., Churazov, E., Sunyaev, R., Forman, W. R., \& Murray, S. S. 2008, Astron. Astrophys., 487, 509

[8] Tueller, J., et al. 2010, Astrophys. J. Suppl., 186, 378 